\title{
A proteomics sample metadata representation for multiomics integration and big data analysis
}

Chengxin Dai', Anja Füllgrabe (1) 2, Julianus Pfeuffer ${ }^{3,4}$, Elizaveta M. Solovyeva (iD 5,6, Jingwen Deng', Pablo Moreno (iD 2, Selvakumar Kamatchinathan ${ }^{2}$, Deepti Jaiswal Kundu², Nancy George (1) 2, Silvie Fexova ${ }^{2}$, Björn Grüning (10 7, Melanie Christine Föll ${ }^{8,9}$, Johannes Griss (1) ${ }^{10}$, Marc Vaudel (1) ${ }^{11}$, Enrique Audain (1) ${ }^{12}$, Marie Locard-Paulet (10) ${ }^{13}$, Michael Turewicz (10) 14,15, Martin Eisenacher (10) 14,15, Julian Uszkoreit (10) 14,15, Tim Van Den Bossche (10) ${ }^{16,17}$, Veit Schwämmle (1) ${ }^{18}$, Henry Webel ${ }^{13}$, Stefan Schulze (10) ${ }^{19}$, David Bouyssié (i) ${ }^{20}$, Savita Jayaram ${ }^{21}$, Vinay Kumar Duggineni ${ }^{21}$, Patroklos Samaras (10) ${ }^{22}$, Mathias Wilhelm (D) ${ }^{22}$, Meena Choi (1) 23, Mingxun Wang24, Oliver Kohlbacher (1) 25,26,27, Alvis Brazma (1) 2, Irene Papatheodorou (10) 2, Nuno Bandeira (1D 24,28, Eric W. Deutsch ${ }^{29}$, Juan Antonio Vizcaíno (10 2, Mingze Bai, ${ }^{1,30 凶,}$ Timo Sachsenberg (10 ${ }^{25 凶}$, Lev I. Levitsky (1) ${ }^{6 凶} \&$ Yasset Perez-Riverol ${ }^{2 凶}$

The amount of public proteomics data is rapidly increasing but there is no standardized format to describe the sample metadata and their relationship with the dataset files in a way that fully supports their understanding or reanalysis. Here we propose to develop the transcriptomics data format MAGE-TAB into a standard representation for proteomics sample metadata. We implement MAGE-TAB-Proteomics in a crowdsourcing project to manually curate over 200 public datasets. We also describe tools and libraries to validate and submit sample metadata-related information to the PRIDE repository. We expect that these developments will improve the reproducibility and facilitate the reanalysis and integration of public proteomics datasets.

\footnotetext{
- We amount of proteomics data in public repositories is growing at an unprecedented rate $^{1,2}$. ProteomeXchange (PX) is a consortium of proteomics resources, including the PRIDE database ${ }^{2}$, PASSEL and PeptideAtlas ${ }^{3}$, MassIVE $^{4}$, jPOST $^{5,6}$, iProX ${ }^{7}$, and Panorama Public ${ }^{8}$. As of July 2021, over 27,000 datasets have been submitted to PX data repositories. PX datasets cover the whole spectrum of protein mass spectrometry (MS) analytical methods and experimental designs, which enable biologists and clinicians to study different aspects of the proteome. In parallel to the generalization of data deposition, reuse of public datasets is becoming increasingly popular. However, thus far, data reuse has largely been limited to
} 
benchmarking studies and applications related to peptide and protein identification, with resources such as PeptideAtlas ${ }^{3}$ and GPMDB $^{9}$ systematically reanalyzing data from $\mathrm{PX}^{10}$. Recently, new efforts like ProteomicsDB ${ }^{11}$, MassiVE.Quant ${ }^{4}$, and Expression Atlas ${ }^{12}$ have started to include reanalyzed quantitative public datasets to present baseline and differential protein expression. However, the scalability and broad reuse of public quantitative experiments have been limited by the lack of sample metadata annotation, which unambiguously associates the samples included in each dataset with the corresponding data files ${ }^{13,14}$.

Since 2012, PX resources have been capturing a general dataset description, including the dataset title, description, instrument, protein modifications included in the search, and submitters/ principal investigators, among other data ${ }^{1}$. The files included in each dataset are, on one hand, the output of the corresponding instrument (e.g., RAW files), and on the other hand, the processed results, which can be represented, e.g., in standard file formats such as mzIdentML ${ }^{15}$ or mzTab ${ }^{16}$. Currently, all PX partners mandate two types of information for each dataset: a general dataset description and the files containing the different required data types. Unfortunately, the experimental design and sample-related information are frequently missing in the datasets or are stored in ad hoc ways and/or formats ${ }^{1}$. Information about the biological samples such as the analyzed organ, tissue, disease, or cell line, and the links between the samples and the corresponding data files are often lacking.

Sample-related metadata and their relationship with the data files are well captured in two widespread file formats called ISA$\mathrm{TAB}^{17}$ and MAGE-TAB (MicroArray Gene Expression Tabular $)^{18}$, which are used in metabolomics and transcriptomics, respectively. As of May 2021, ArrayExpress has stored over 74,000 functional genomics datasets in the MAGE-TAB format ${ }^{18,19}$. In both formats, a tab-delimited file is used to annotate the sample metadata and link the metadata to the corresponding data files. While MAGE-TAB was originally designed for microarray experiments, it has been successfully adapted to high-throughput RNA-sequencing and single-cell RNA-Seq experiments ${ }^{20}$.

Here we introduce an extension and implementation of the MAGE-TAB format for proteomics (MAGE-TAB-Proteomics). The format has been developed in collaboration with the Proteomics Standards Initiative (PSI), the organization in charge of developing open-standard formats in the field ${ }^{21}$. We have also developed general guidelines about what information needs to be encoded in MAGE-TAB to improve the reproducibility and enable the reanalysis of proteomics datasets. In addition, we have crowdsourced the annotation of over 200 existing public datasets according to these guidelines, covering different analytical methods and experimental designs. Finally, we have developed an ecosystem of tools to validate MAGE-TAB-Proteomics files and integrate the metadata in the PRIDE database, the most popular $\mathrm{PX}$ resource. The full specification document describing all aspects of MAGE-TAB-Proteomics version 1.0, the current implementations, as well as application examples, is available at the PSI website (https://psidev.info/magetab).

Repurposing MAGE-TAB for proteomics. MAGE-TAB encodes the sample metadata annotations and the information linking the metadata to the corresponding data files in two different files: the Investigation Description Format (IDF) and the Sample and Data Relationship Format (SDRF). In the following, we describe how we adapted these formats to the specific needs of proteomics.

Providing study-description information in IDF. The IDF file contains information describing the study, including, e.g., authors/submitters, protocols, and publications (Supplementary Note 1). The IDF format contains a series of key/value pairs, where each key represents a different property. For example, "Experiment Description" should be followed by a free-text description of the experiment (which would be the value). Most of the fields can contain more than one value, so that multiple values (e.g., multiple-analysis software tools) can be defined in a single IDF file. Since 2012, PX dataset descriptions are provided using PX XML (http://proteomecentral.proteomexchange.org/ schemas/proteomeXchange-1.4.0.html), an XML file format that captures equivalent information to the ones included in IDF, making both files easily exchangeable (Supplementary Note 1). Therefore, we developed the IDF component of MAGE-TAB based on the existing PX XML format.

Linking samples to data files with SDRF. SDRF is a tabdelimited file that describes the samples and allows their mapping to the data files ${ }^{1}$. As shown in Fig. 1a, SDRF includes the annotation of (i) biological sample metadata; (ii) the relationships between samples and data files; (iii) (technical) metadata of RAW data files; and (iv) the variables under study (called factor values). Each row in an SDRF file corresponds to one relationship between a sample and a data file (an MS RAW file or a channel included in a given RAW file in the case of labeling-based proteomics). Each column corresponds to an attribute/property of the sample or the file, and the value in each cell is the specific value of the property (Fig. 1a).

All the properties in the SDRF must be encoded as ontology terms, whereas the values of the properties can be encoded as ontology terms, numerical values, or free text. To facilitate the annotation, validation, and processing of SDRF files, a list of ontologies has been defined that can be used for encoding each property. For example, most of the sample properties are included in the Experimental Factor Ontology ${ }^{22}$ (EFOhttps://www.ebi.ac.uk/efo/), while most of the data-file properties are included in PSI-MS-controlled vocabulary (https:// www.ebi.ac.uk/ols/ontologies/ms) and the PRIDE ontology (https://www.ebi.ac.uk/ols/ontologies/pride).

Each sample in an SDRF file has a unique identifier (source name), and every sample property is encoded using the prefix characteristics (e.g., characteristics [organism part]). Each data file also has a unique identifier (assay name), and every file property has the prefix comment (e.g., comment[instrument], comment[fraction identifier]). Finally, the variables under study must be specified with the prefix factor value (e.g., factor value[tissue]). The MAGE-TABProteomics specification defines the minimum information that should be provided for every sample and data file (https:// github.com/bigbio/proteomics-metadata-standard/raw/master/psidocument/HUPO-PSI-MAGETAB-Proteomics_latest.docx). For all proteomics experiments, the following properties must be provided: organism, organism part, and biological replicate accession. For every data file, the following properties are required: fraction identifier, technical replicate accession, label (in the case of labeling methods), and data-file name. Biological and technical replicates should be explicitly included using the terms characteristics[biological replicate] and comment[technical replicate], respectively (Fig. 1a). The biological replicate field is considered a property of the samples, whereas the technical replicate is considered a property of the data files.

A second category of fields includes information that is not mandatory but recommended. Each PX repository can define which of the recommended fields must be provided in their resource, depending on the experiment types. The current PX templates request the submitters to provide the following properties for every data file: instrument model, cleavage agent, 


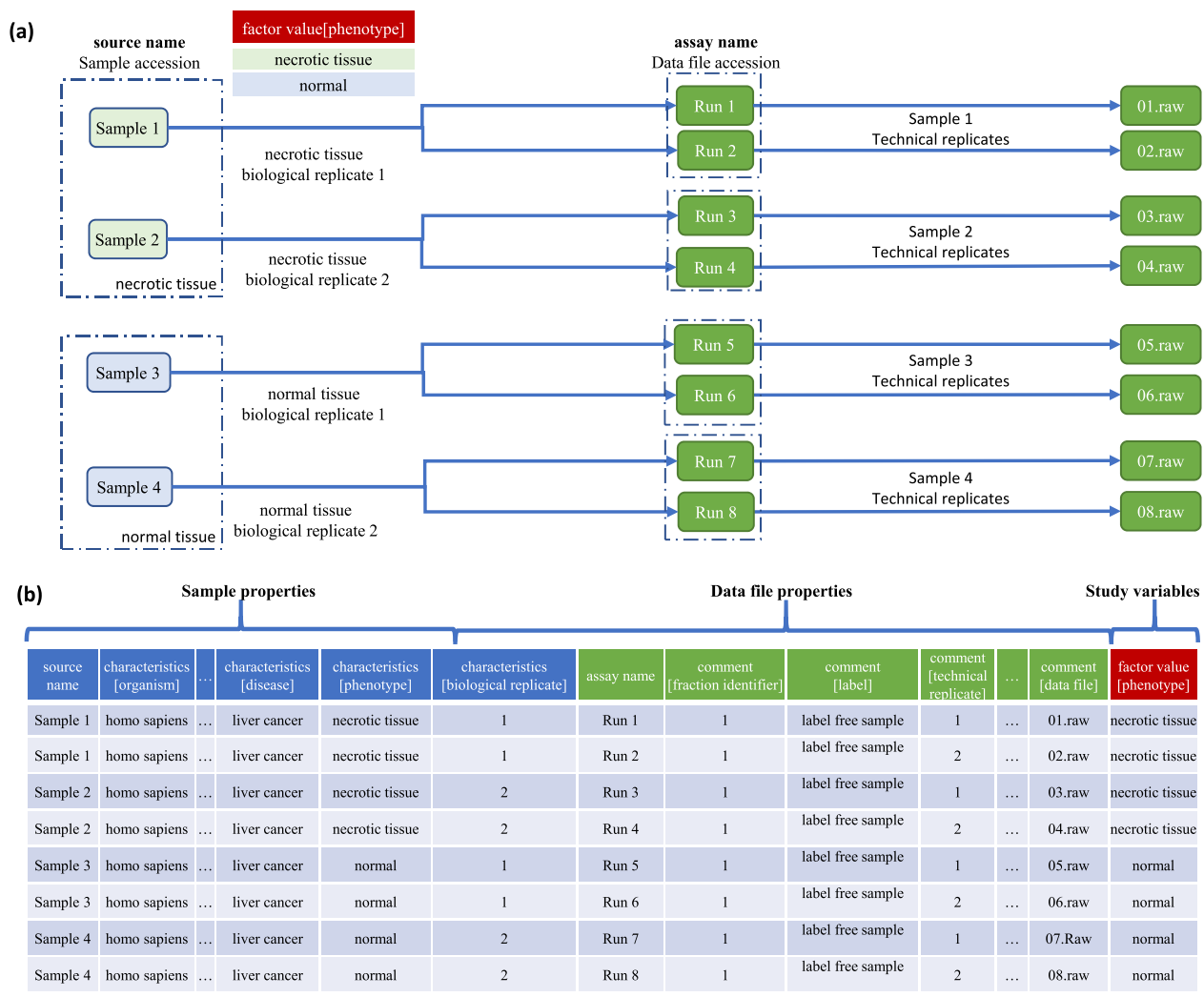

Fig. 1 SDRF-Proteomics representation for a label-free-based experiment without fractionation. a Experimental design, including two biological replicates and two technical replicates per biological replicate. The biological and technical replicates are defined by the variable under study (e.g., phenotype). b The SDRF tab-delimited file, including the three main sections highlighted: sample metadata, data file properties, and the variables under study (factor values).

fragment-mass tolerance, precursor-mass tolerance, and mass modifications (e.g., post-translational modifications (PTMs) and artifactual modifications considered in the analysis). Most of the values of these properties can be encoded as a combination of multiple key/value pairs (e.g., methionine oxidation can be specified as $\mathrm{AC}=\mathrm{UNIMOD}: 35 ; \mathrm{NT}=$ Oxidation; $\mathrm{MT}=$ Variable; $\mathrm{TA}=\mathrm{M}$ ). We believe that this represents the minimum set of information that is necessary for practical terms for understanding and re-using MS-based proteomics datasets. Furthermore, other properties such as labeling can be provided. Importantly, the set of mandatory properties can be readily expanded in case the PX community decides to extend its metadata requirements in the future. For now, we have defined additional templates (Supplementary Table 1), which form a set of recommended properties required per experiment type. Submitters can use the template corresponding to their experiment to streamline annotation. For example, the cell line (characteristics[cell line]) is a recommended metadata item for cell-line experiments; for every human dataset, the disease under study should be provided in the field characteristics[disease] and the control samples should be labeled with the value "normal".

Multiplexing and fractionation. Transcriptomics datasets typically show a one-to-one relationship between each sample and data file. While this is also the case for some proteomics data, two popular experimental designs in proteomics-sample multiplexing and fractionation-follow different patterns (Fig. 2).

In multiplexed quantitative experiments (e.g., based on tandem mass tag (TMT) labeling), multiple samples can be related to the same data file (Fig. 2a). In these cases, the data-file properties should be repeated for each sample including all the properties (e.g., instrument). The different samples included in the same data file can be encoded using the relevant property labels (e.g., comment[label] $=$ TMT128N).

When fractionation is used, the sample information should be repeated for each data file. A property called fraction identifier is used to make clear which fractions correspond to a given data file (e.g., comment[fraction identifier] =1).

These features make MAGE-TAB-Proteomics highly flexible and applicable to complex experimental designs involving both fractionation and multiplexing (Fig. 2b). While the duplication of information can be perceived as redundant, it enables a streamlined data reanalysis because each row/line of the SDRF can be processed individually. In addition, it facilitates metaanalysis with simple operations such as merging different SDRFs coming from different datasets or splitting an SDRF for a given dataset by a specific property of the data file or the sample.

\section{Crowdsourcing annotations of public proteomics datasets}

Crowdsourcing has been successfully applied to address key problems in bioinformatics ${ }^{23}$. The European Bioinformatics Community for Mass Spectrometry (EuBIC-MS ${ }^{24}$, https://eubicms.org) set up a collective effort to annotate existing PX datasets using MAGE-TAB-Proteomics ${ }^{14}$. Volunteers from multiple institutes joined the task of annotating, discussing, and improving the SDRF format, openly and collaboratively. Each annotator created an issue in GitHub about a project of interest, forked the main project repository (https://github.com/bigbio/proteomicsmetadata-standard), and annotated the corresponding dataset locally in their computers. Then, a pull request was submitted to include the added annotations. Prior to approval, an independent group of reviewers checked that the proposed SDRF conformed 
(a)

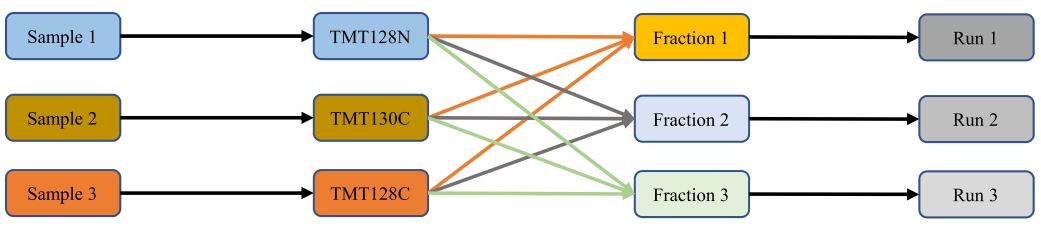

(b)

\begin{tabular}{|c|c|c|c|c|c|c|c|c|c|c|}
\hline $\begin{array}{l}\text { source } \\
\text { name }\end{array}$ & $\begin{array}{l}\text { characteristics } \\
\text { [organism] }\end{array}$ & $\begin{array}{l}\text { characteristics } \\
\text { [disease] }\end{array}$ & $\begin{array}{l}\text { characteristics } \\
\text { [tumor stage] }\end{array}$ & & assay name & comment[data file] & $\begin{array}{l}\text { comment } \\
\text { [fraction } \\
\text { identifier] }\end{array}$ & $\begin{array}{l}\text { comment } \\
\text { [label] }\end{array}$ & & $\begin{array}{c}\text { factor value } \\
\text { [tumor stage] }\end{array}$ \\
\hline Sample 1 & Homo sapiens & $\begin{array}{l}\text { Breast Invasive } \\
\text { Carcinoma }\end{array}$ & Stage IIA & $\cdots$ & run 1 & BL_f01.raw & 1 & TMT128N & $\cdots$ & Stage IIA \\
\hline Sample 2 & Homo sapiens & $\begin{array}{l}\text { Breast Invasive } \\
\text { Carcinoma }\end{array}$ & Stage III & $\ldots$ & run 1 & BL_f01.raw & 1 & TMT130C & $\ldots$ & Stage III \\
\hline Sample 3 & Homo sapiens & $\begin{array}{l}\text { Breast Invasive } \\
\text { Carcinoma }\end{array}$ & Stage IIIA & $\cdots$ & run 1 & BL_f01.raw & 1 & TMT128C & $\ldots$ & Stage IIIA \\
\hline Sample 1 & Homo sapiens & $\begin{array}{l}\text { Breast Invasive } \\
\text { Carcinoma }\end{array}$ & Stage IIA & $\cdots$ & run 2 & BL_f02.raw & 2 & TMT128N & $\cdots$ & Stage IIA \\
\hline Sample 2 & Homo sapiens & $\begin{array}{l}\text { Breast Invasive } \\
\text { Carcinoma }\end{array}$ & Stage III & $\ldots$ & run 2 & BL_f02.raw & 2 & TMT130C & $\cdots$ & Stage III \\
\hline Sample 3 & Homo sapiens & $\begin{array}{l}\text { Breast Invasive } \\
\text { Carcinoma }\end{array}$ & Stage IIIA & $\cdots$ & run 2 & BL_f02.raw & 2 & TMT128C & $\cdots$ & Stage IIIA \\
\hline Sample 1 & Homo sapiens & $\begin{array}{l}\text { Breast Invasive } \\
\text { Carcinoma }\end{array}$ & Stage IIA & $\cdots$ & run 3 & BL_f03.raw & 3 & TMT128N & $\cdots$ & Stage IIA \\
\hline Sample 2 & Homo sapiens & $\begin{array}{l}\text { Breast Invasive } \\
\text { Carcinoma }\end{array}$ & Stage III & $\cdots$ & run 3 & BL_f03.raw & 3 & TMT130C & $\cdots$ & Stage III \\
\hline Sample 3 & Homo sapiens & $\begin{array}{l}\text { Breast Invasive } \\
\text { Carcinoma }\end{array}$ & Stage IIIA & $\cdots$ & run 3 & BL_f03.raw & 3 & TMT128C & $\cdots$ & Stage IIIA \\
\hline
\end{tabular}

Fig. 2 SDRF-Proteomics file for an experiment combining TMT labeling and sample fractionation. a TMT experimental design with three samples and three fractions. $\mathbf{b}$ SDRF representation for a TMT experiment with three samples and three fractions resulting in nine rows where samples are repeated for each fraction and data-file information is repeated for each labeling channel, which is encoded using the property comment[label].

to the guidelines. This collaborative peer-review system allowed the identification of potential issues in the file format, which is now compatible with the main MS experiment types. Additionally, a file validator was developed to automatically perform a semantic and structural validation of created SDRF files (see next section for details).

As of July 2021, over 200 public datasets have been annotated, covering a broad spectrum of organisms, enrichment/fractionation strategies, quantification approaches, and data-acquisition methods (Fig. 3a). For most multiplexed experiments available on $\mathrm{PX}$, the sample-to-label assignment was not specified by the authors since it was not possible to map the channel to the associated sample in a standardized format. The lack of such essential experimental design information precludes the reprocessing and reproduction of quantitative results. As a consequence, such datasets are underrepresented in the collection of annotated datasets. This highlights the urgent need for systematic and standardized metadata annotations. To support future annotation efforts, Table 1 shows gold-standard annotated datasets from PX that can be utilized as examples when creating an SDRF file for various experimental designs (e.g., label-free quantification, multiplex TMT, stable isotope labeling by amino acids in cell culture (SILAC), affinity purification-mass spectrometry (AP-MS), data-independent acquisition (DIA), and phosphopeptide enrichment).

\section{The MAGE-TAB-proteomics toolbox}

To facilitate automatic data analysis and reuse, we developed a set of software libraries that enable the validation and conversion from MAGE-TAB-Proteomics format to parameter files (Fig. 3b). For transcriptomics data, the main library to validate a MAGE$\mathrm{TAB}$ file is the Bio-MAGETAB Perl package (https:// metacpan.org/release/Bio-MAGETAB). In principle, BioMAGETAB can also be used to validate MAGE-TABProteomics files because they are valid MAGE-TAB files.
However, Bio-MAGETAB cannot perform extra validations specific to proteomics data, especially the SDRF-Proteomics rules. To enable the validation of the SDRF-Proteomics of MAGE-TABProteomics files, we implemented two libraries in Java and Python (see below). After validation, parameters such as cleavage agents, post-translational modifications, and mass tolerances (precursor and fragment) are translated into MaxQuant and OpenMS parameters in their corresponding configuration files.

The newly developed Python package, called sdrf-pipelines (https://github.com/bigbio/sdrf-pipelines), enables the validation of the structure and also of the semantic rules applied to SDRF. The sdrf-pipelines package can be installed from different package managers like BioConda ${ }^{25}$ or BioContainers ${ }^{26}$. It validates the files according to the different experiments and data types, as defined in the templates. For example, if the template corresponds to a human dataset, the software validates that the sample metadata comply with the human template (organism, disease, ancestry, etc.). In addition, sdrf-pipelines allows users to convert SDRF files to configuration/input files of other popular proteomics analysis tools such as MaxQuant ${ }^{27}$, OpenMS ${ }^{28}$, and MSstats $^{29}$, to facilitate the automation of dataset reanalyses (https://github.com/bigbio/proteomics-metadata-standard/wiki).

The jSDRF Java library (https://github.com/bigbio/jsdrf) we developed also enables the validation of SDRF files. For example, the library can validate that the SDRF contains each sample and data-file relationship, labeling information, fraction identifiers, and the sample and data accessions. It also includes a generic data model that can be used by Java applications to validate and handle SDRF-Proteomics files.

While the PX resources have not yet developed any tool to create SDRF-Proteomics files, existing tools for transcriptomics MAGE$\mathrm{TAB}$ and ISA-TAB can be used to facilitate the process. At the moment of writing, we recommend OntoMaton ${ }^{30}$ (https:// github.com/bigbio/proteomics-metadata-standard/wiki/AnnotatingSample-terms-using-OntoMaton), which allows the annotation of Google spreadsheets using ontology terms coming from the Ontology 

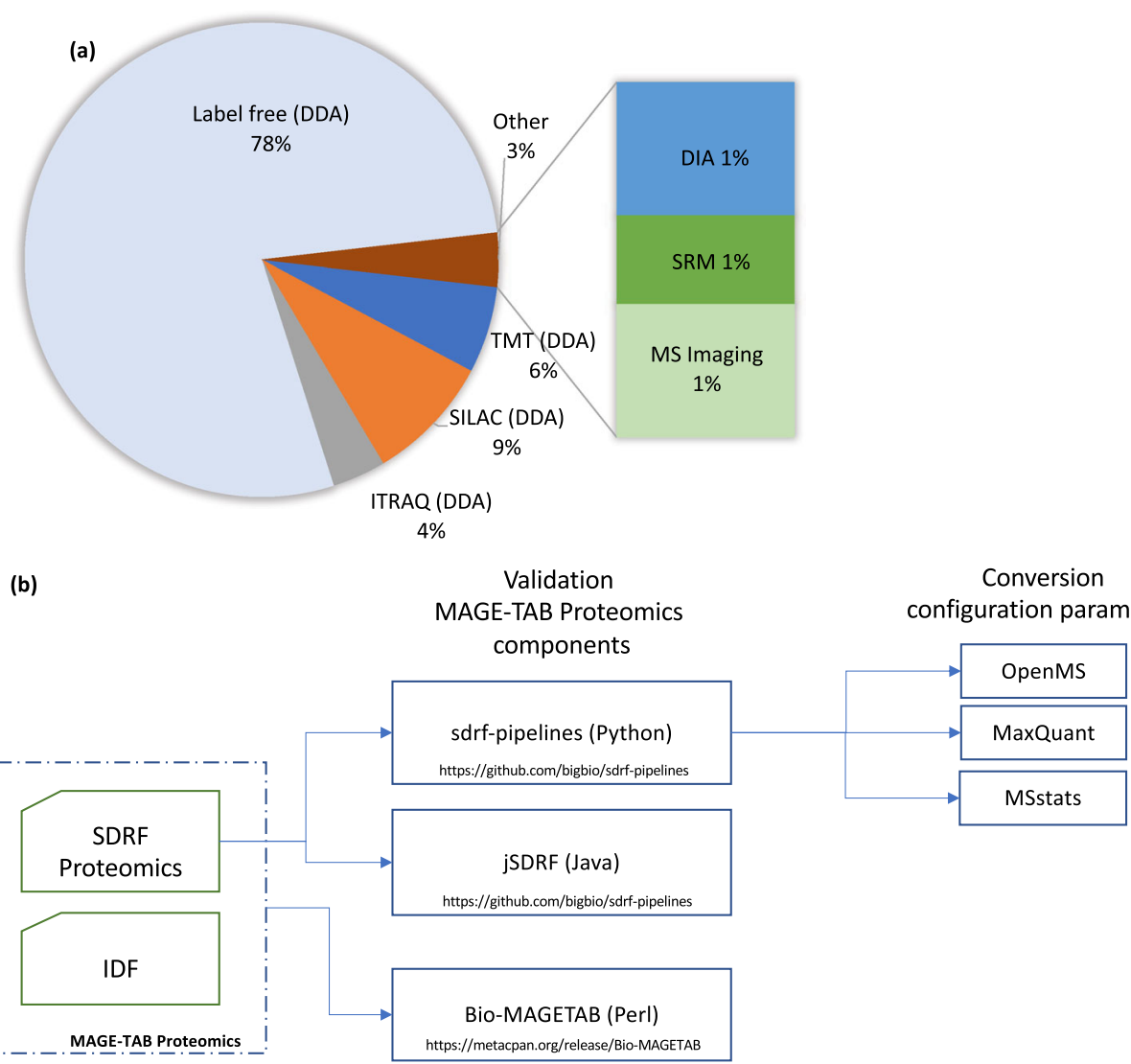

Fig. 3 Using MAGE-TAB-Proteomics for dataset annotations. a Quantification and data-acquisition methods used in the public datasets that have been annotated with MAGE-Tab-Proteomics by July 2021. b Tools and libraries for the validation and conversion of MAGE-TAB-Proteomics files.

Table 1 Examples of MAGE-TAB-Proteomics files.

\section{Dataset type}

Label-free

TMT, CPTAC dataset not in PX

SILAC

Phospho-proteomics

Label-free, multiple fragmentation modes and various enzymes

AP-MS interactomics

TMT

Label-free

DIA

Metabolomics

\section{Accession code/hyperlink}

PXD008934

https://cptac-data-portal.georgetown.edu/ study-summary/SO29 36 PXD006877

PXD006482

PXD010154

PXD018117

PXD017710

PXD004242

PXD003539

MSV000086206 [https://doi.org/ doi:10.25345/C5HVOS]

\section{MAGE-TAB}

https://github.com/bigbio/proteomics-metadatastandard/tree/master/annotated-projects/PXD008934 https://github.com/bigbio/proteomics-metadatastandard/tree/master/annotated-projects/PMID33212010 https://github.com/bigbio/proteomics-metadatastandard/tree/master/annotated-projects/PXD006877 https://github.com/bigbio/proteomics-metadatastandard/tree/master/annotated-projects/PXD006482 https://github.com/bigbio/proteomics-metadatastandard/tree/master/annotated-projects/PXD010154 https://github.com/bigbio/proteomics-metadatastandard/tree/master/annotated-projects/PXD018117 https://github.com/bigbio/proteomics-metadatastandard/tree/master/annotated-projects/PXD017710 https://github.com/bigbio/proteomics-metadatastandard/tree/master/annotated-projects/PXD004242 https://github.com/bigbio/proteomics-metadatastandard/tree/master/annotated-projects/PXD003539 https://github.com/bigbio/proteomics-metadatastandard/tree/master/annotated-projects/ MSV000086206
Lookup Service $(\mathrm{OLS})^{31}$. Submitters can search ontology terms in OLS and add them to the sample-property columns. The Google spreadsheets provide the functionalities to copy ontology terms across samples, and to add or remove columns and samples. New versions of the tool will include the possibility to add protein modifications, online validation, and loading existing templates among others.

\section{Submitting annotated proteomics datasets to the PRIDE database}

Datasets are standardly submitted to PRIDE using the PX submission tool, a desktop application that guides the users through a set of steps to construct the submission and finally performs the transfer of the data files ${ }^{2}$ (Fig. 4). The information annotated by the submitter is encoded into a submission.px file. As described 


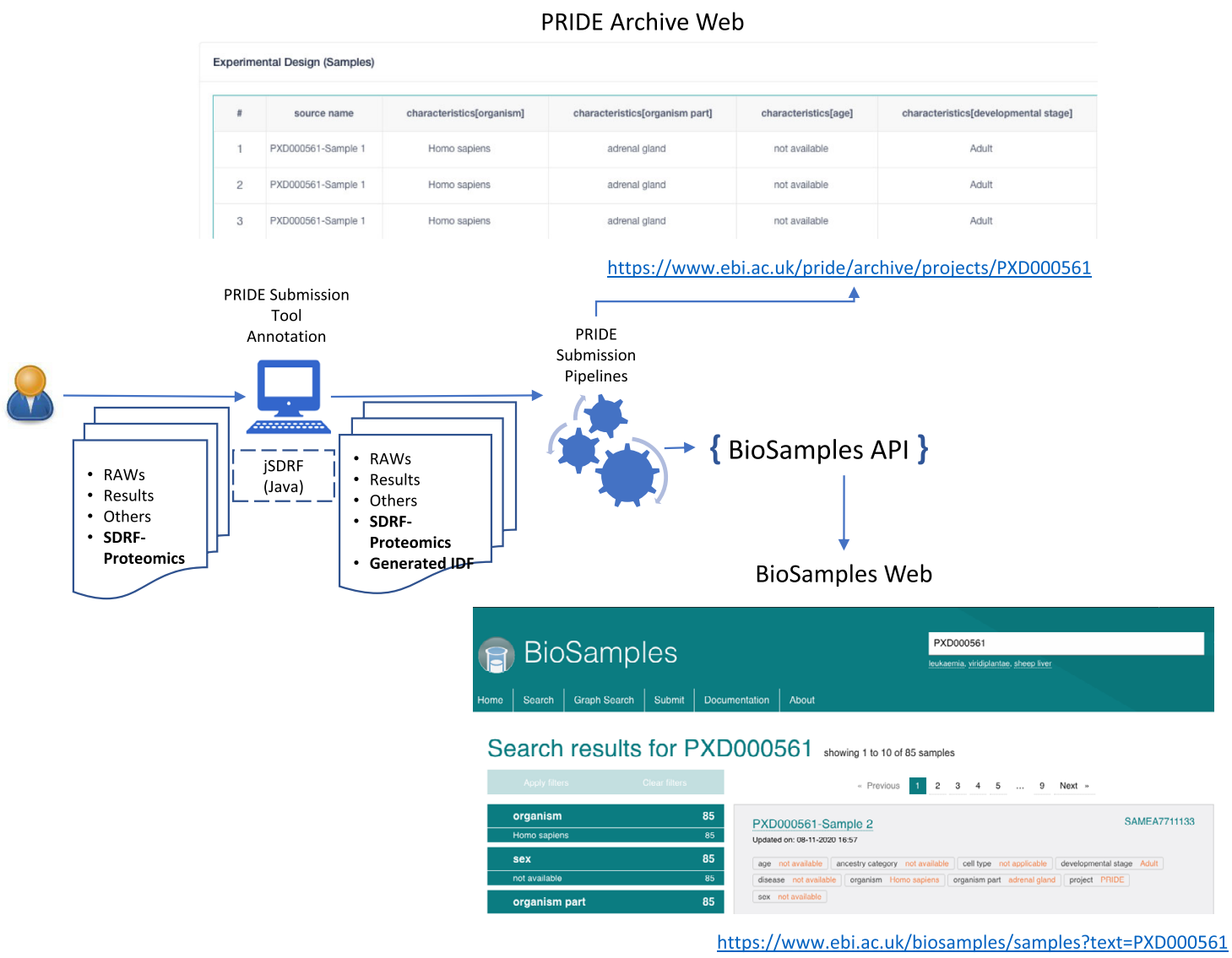

Fig. 4 PRIDE database-submission workflow supporting IDF and SDRF files. The IDF file is automatically generated during submission; the SDRF file can be provided by the user in the PRIDE Submission Tool and is automatically validated in the submission pipeline. The sample information is shown on the web page of each PRIDE dataset and submitted to the EBI BioSample database, which assigns a unique accession to each sample. Shown are representative PRIDE and BioSamples outputs for the dataset PXD000561.

above, this PX XML file is highly similar to the MAGE-TABProteomics IDF file. Therefore, the PX XML file is now automatically converted to IDF using PRIDE internal pipelines, once the submission is received and processed.

Since May 2021, the PX submission tool accepts SDRF files. The SDRF file can be created externally as described in the previous section and submitted with the dataset. It is then recognized as an 'EXPERIMENT DESIGN' file type and validated using the jSDRF library (Fig. 4). The PRIDE internal pipelines create MAGE-TAB-Proteomics files containing the automatically generated IDF and the user-provided SDRF. Then, the metadata assigned to each sample is automatically submitted to the BioSamples database ${ }^{32}$. A BioSample accession number is created for each sample in the experiment to enable the linking between samples included in multi-omics datasets. The PRIDE web interface presents for each dataset the associated sample metadata table. In addition, the sample metadata are indexed by PRIDE, allowing users to locate and link samples and experiments within the vast number of public datasets.

\section{Conclusions and future perspectives}

Resources such as MassIVE.Quant, ExpressionAtlas, and ProteomicsDB have recently started to systematically reanalyze proteomics public quantitative datasets ${ }^{4,11,19}$. However, the lack of sample metadata that allow associating sample properties with data files makes this task complex and unscalable. Capturing metadata is challenging, since this generic term ranges from concepts such as the title of the experiment to sample-related information, to technical details like the instrument-configuration parameters used for data acquisition. To represent these different levels of proteomics metadata, MAGE-TAB-Proteomics builds upon a popular and flexible data format adopted from transcriptomics that relies on IDF and SDRF files. The IDFProteomics format closely resembles the PX XML format that PX resources have long been used to capture dataset information. The SDRF-Proteomics format has a schema and data model that allows submitters to provide the minimum information about the samples as well as a more complete record of their sample and RAW file metadata. The SDRF templates define the minimum information PX partners have agreed upon, which should be provided for each submitted dataset. Additionally, the file format enables users and submitters to describe in much higher detail the sample, sample-processing steps, and the RAW files. Owing to this flexibility, MAGE-TAB is compatible with a wide range of experimental approaches (Table 1) and can be easily adapted to changing metadata requirements in the future. Importantly, the MAGE-TAB-Proteomics project is supported by the worldleading proteomics databases in PX. Due to the gradual and iterative implementation of MAGE-TAB-Proteomics into PX submission pipelines and related tools, the adoption of the standard will not create a major additional burden for users. To support new users, we have created a tutorial in GitHub (https:// github.com/bigbio/proteomics-metadata-standard/wiki) and a video that introduces the file format (https://www.youtube.com/ watch?v=TMDu_yTzYQM).

MAGE-TAB-Proteomics will facilitate the integration and annotation of proteomics studies, thereby enhancing their 
discoverability, reproducibility, and reusability. It holds great potential to facilitate the use of standardized workflows for the automated reanalysis of currently available and future annotated public proteomics datasets. This will increase the inherent value of proteomics datasets by making them more amenable for largescale meta-analysis, systems biology, and multiomics projects that can take advantage of large hardware resources in cloud-based environments. The proposed standard metadata representation will also facilitate the development of common submission systems to streamline the deposition of multiomics datasets to resources for different types of omics data. It may even foster the creation of new multiomics resources, enabling submission, validation, and visualization of multi-omics data in one place ${ }^{33,34}$. As an initial step in this direction, the PRIDE group has started to reanalyze proteomics datasets represented in SDRF and integrates them into the multiomics resource Expression Atlas (e.g., https:// www.ebi.ac.uk/gxa/experiments/E-PROT-18/Results).

Expanding the MAGE-TAB-Proteomics format to additional use cases and more types of MS-based experiments, including metaproteomics and xenograft proteomics experimental designs, is currently under discussion (https://github.com/bigbio/ proteomics-metadata-standard/blob/master/sdrf-proteomics/usecases-under-development.adoc). Researchers interested in use cases that are currently not supported are encouraged to bring them to our attention by creating an issue in GitHub using the above hyperlink. Analogous to the implementation process of other PSI formats, we aim to engage software tools and developers to support the MAGE-TAB-Proteomics format as an input file for data processing and as output file for the datasetsubmission process. Notably, the MAGE-TAB-Proteomics format has already triggered interest in the MS metabolomics field. Firstmetabolomics data have already been annotated using MAGETAB-Proteomics (Table 1) and more metabolomics datasets coming from the ReDU resource ${ }^{35}$ have been added to the annotation repository, underscoring the open, collaborative, and community-driven approach of this project. In this spirit, we invite all interested parties to join the MAGE-TAB-Proteomics initiative.

\section{Data availability}

The annotated datasets generated in this study are provided in GitHub (https:// github.com/bigbio/proteomics-metadata-standard/tree/master/annotated-projects). The raw data corresponding to the example datasets shown in Table 1 are available under the accession codes PXD008934, PXD006877, PXD006482, PXD010154, PXD018117, PXD017710, PXD004242, PXD003539, MSV000086206 [https://doi.org/doi:10.25345/ C5HV0S]. The raw data of the TMT/CPTAC dataset are available at https://cptac-dataportal.georgetown.edu/study-summary/S029.

\section{Code availability}

General documentation and tutorials as well as a script to generate IDF files are provided in Github (https://github.com/bigbio/proteomics-metadata-standard); however, please note that IDF files are also automatically generated during the PRIDE submission process. The code of the sdrf-pipelines tool developed in this study is deposited in GitHub (https://github.com/bigbio/sdrf-pipelines). The jSDRF Java library is available at https://github.com/bigbio/jsdrf. The Bio-MAGETAB Perl package is available at https:// metacpan.org/release/Bio-MAGETAB.

Received: 19 May 2021; Accepted: 16 September 2021; Published online: 06 October 2021

\section{References}

1. Deutsch, E. W. et al. The ProteomeXchange consortium in 2020: enabling 'big data' approaches in proteomics. Nucleic Acids Res. 48, D1145-D1152 (2020). ProteomeXchange consortium manuscript including the ecosystem to discuss data sharing policies and formats in proteomics.

2. Perez-Riverol, Y. et al. The PRIDE database and related tools and resources in 2019: improving support for quantification data. Nucleic Acids Res. 47,
D442-D450 (2019). PRIDE database manuscript, which has led the development and integration of MAGE-TAB-Proteomics with other EMBL-EBI resources such as BioSamples and Expression Atlas.

3. Deutsch, E. W. The peptideatlas project. Methods Mol. Biol. 604, 285-296 (2010)

4. Choi, M. et al. MassIVE.quant: a community resource of quantitative mass spectrometry-based proteomics datasets. Nat. Methods 17, 981-984 (2020).

5. Watanabe, Y., Yoshizawa, A. C., Ishihama, Y. \& Okuda, S. The jPOST repository as a public data repository for shotgun proteomics. Methods Mol. Biol. 2259, 309-322 (2021).

6. Moriya, Y. et al. The jPOST environment: an integrated proteomics data repository and database. Nucleic Acids Res. 47, D1218-D1224 (2019).

7. Ma, J. et al. iProX: an integrated proteome resource. Nucleic Acids Res. 47, D1211-D1217 (2019).

8. Sharma, V. et al. Panorama Public: a public repository for quantitative data sets processed in skyline. Mol. Cell Proteom. 17, 1239-1244 (2018).

9. Craig, R., Cortens, J. P. \& Beavis, R. C. Open source system for analyzing validating, and storing protein identification data. J. Proteome Res. 3, 1234-1242 (2004).

10. Perez-Riverol, Y., Alpi, E., Wang, R., Hermjakob, H. \& Vizcaino, J. A. Making proteomics data accessible and reusable: current state of proteomics databases and repositories. Proteomics 15, 930-949 (2015).

11. Samaras, P. et al. ProteomicsDB: a multi-omics and multi-organism resource for life science research. Nucleic Acids Res. 48, D1153-D1163 (2020).

12. Papatheodorou, I. et al. Expression Atlas update: from tissues to single cells. Nucleic Acids Res. 48, D77-D83 (2020).

13. Griss, J., Perez-Riverol, Y., Hermjakob, H. \& Vizcaino, J. A. Identifying novel biomarkers through data mining-a realistic scenario? Proteom. Clin. Appl. 9, 437-443 (2015).

14. Perez-Riverol, Y. \& European Bioinformatics Community for Mass Spectrometry. Toward a sample metadata standard in public proteomics repositories. J. Proteome Res. 19, 3906-3909 (2020).

15. Vizcaino, J. A. et al. The mzIdentML data standard version 1.2, supporting advances in proteome informatics. Mol. Cell Proteom. 16, 1275-1285 (2017)

16. Griss, J. et al. The mzTab data exchange format: communicating massspectrometry-based proteomics and metabolomics experimental results to a wider audience. Mol. Cell Proteom. 13, 2765-2775 (2014). Manuscript describing the mzTab file format, which contains the actual expression values in proteomics and may in the future be linked to MAGE-TABProteomics in the PRIDE database.

17. Gonzalez-Beltran, A., Maguire, E., Sansone, S. A. \& Rocca-Serra, P. linkedISA semantic representation of ISA-Tab experimental metadata. BMC Bioinforma. 15, S4 (2014).

18. Rayner, T. F. et al. A simple spreadsheet-based, MIAME-supportive format for microarray data: MAGE-TAB. BMC Bioinforma. 7, 489 (2006). Original publication of MAGE-TAB for transcriptomics experiments defining the principles of the file format and data model.

19. Athar, A. et al. ArrayExpress update - from bulk to single-cell expression data. Nucleic Acids Res. 47, D711-D715 (2019).

20. Fullgrabe, A. et al. Guidelines for reporting single-cell RNA-seq experiments. Nat. Biotechnol. 38, 1384-1386 (2020). Recent extension of the MAGE-TAB for single cell RNA expression datasets.

21. Deutsch, E. W. et al. Proteomics standards initiative: fifteen years of progress and future work. J. Proteome Res. 16, 4288-4298 (2017).

22. Malone, J. et al. Modeling sample variables with an Experimental Factor Ontology. Bioinformatics 26, 1112-1118 (2010).

23. Good, B. M. \& Su, A. I. Crowdsourcing for bioinformatics. Bioinformatics $\mathbf{2 9}$, 1925-1933 (2013).

24. Ashwood, C. et al. Proceedings of the EuBIC-MS 2020 Developers' Meeting. EuPA Open Proteom. 24, 1-6 (2020).

25. Gruning, B. et al. Bioconda: sustainable and comprehensive software distribution for the life sciences. Nat. Methods 15, 475-476 (2018).

26. Bai J., et al. BioContainers Registry: searching bioinformatics and proteomics tools, packages, and containers. J. Proteome Res., 20, 2056-2061 (2021).

27. Sinitcyn, P. et al. MaxQuant goes Linux. Nat. Methods 15, 401 (2018).

28. Pfeuffer, J. et al. OpenMS - A platform for reproducible analysis of mass spectrometry data. J. Biotechnol. 261, 142-148 (2017).

29. Choi, M. et al. MSstats: an R package for statistical analysis of quantitative mass spectrometry-based proteomic experiments. Bioinformatics 30, 2524-2526 (2014)

30. Maguire, E., Gonzalez-Beltran, A., Whetzel, P. L., Sansone, S. A. \& RoccaSerra, P. OntoMaton: a bioportal powered ontology widget for Google Spreadsheets. Bioinformatics 29, 525-527 (2013).

31. Perez-Riverol Y., et al. OLS Client and OLS Dialog: open source tools to annotate Public Omics Datasets. Proteomics 17, 1700244 (2017).

32. Courtot, M. et al. BioSamples database: an updated sample metadata hub. Nucleic Acids Res. 47, D1172-D1178 (2019).

33. Sarkans, U. et al. From ArrayExpress to BioStudies. Nucleic Acids Res. 49, D1502-D1506 (2021). 
34. Perez-Riverol, Y. et al. Discovering and linking public omics data sets using the Omics Discovery Index. Nat. Biotechnol. 35, 406-409 (2017).

35. Jarmusch, A. K. et al. ReDU: a framework to find and reanalyze public mass spectrometry data. Nat. Methods 17, 901-904 (2020).

36. Mertins, P. et al. Proteogenomics connects somatic mutations to signalling in breast cancer. Nature 534, 55-62 (2016).

\section{Acknowledgements}

YPR, SK, DJK, and JAV would like to acknowledge funding from the Wellcome Trust grant number 208391/Z/17/Z and EMBL core funding. MLP is supported financially by the Novo Nordisk Foundation (Grant agreement NNF14CC0001). MT and TS are supported by de.NBI, a project of the German Federal Ministry of Education and Research (BMBF) [grant number FKZ 031 A 534 A and FKZ 031 A 535 A]. ME and JU are members of the Center for Protein Diagnostics (PRODI), a grant from the Ministry of Innovation, Science, and Research of North-Rhine Westphalia, Germany. TVDB is supported by the Research Foundation-Flanders (SB grant 1S90918N). EWD acknowledges NIGMS grants R01GM087221, R24GM127667, and NSF grants 1933311 and 1922871. SS was supported by the NSF grant 1817518. CD and MB are supported by the National Key Research and Development Program of China (2017YFC0908404, 2017YFC0908405) and the Natural Science Foundation of Chongqing, China (cstc2018jcyjAX0225). Funds for the overall project were also made available by an ELIXIR Implementation Study. LIL and EMS are supported by the Russian Basic Science Foundation (grant \#18-29-13015)

\section{Author contributions}

Y.P.R, C.D, J.D, E.M.S, D.J.K, M.C.F, J.G, M.V, E.A, M.L.P, M.T, M.E, J.U, T.V.D.B, V.S, H.W, S.S, D.B, S.J, V.K.D, P.S, M.W, A.F, N.G, S.F, M.C, M.B, O.K, A.B, I.P, N.B, E.W.D, and J.A.V contributed to the definition of the specification and annotated the GitHub public proteomics datasets. Y.P.R, C.D, J.P, P.M, S.K, B.G, L.I.L, and T.S developed the MAGE-TAB for proteomics libraries and integration with the PRIDE database. N.B, M.C, E.W.D, J.A.V, and Y.P.R introduced the specification to HUPO-PSI and ProteomeXchange consortium. All authors contributed to the writing and review of the present paper.

\section{Competing interests}

The authors declare no competing interests.

\section{Additional information}

Supplementary information The online version contains supplementary material available at https://doi.org/10.1038/s41467-021-26111-3.

Correspondence and requests for materials should be addressed to Mingze Bai, Timo Sachsenberg, Lev I. Levitsky or Yasset Perez-Riverol.

Peer review information Nature Communications thanks Ruedi Aebersold and Yunping $\mathrm{Zhu}$ for their contribution to the peer review of this work.

Reprints and permission information is available at http://www.nature.com/reprints

Publisher's note Springer Nature remains neutral with regard to jurisdictional claims in published maps and institutional affiliations.

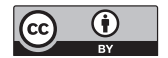

Open Access This article is licensed under a Creative Commons Attribution 4.0 International License, which permits use, sharing, adaptation, distribution and reproduction in any medium or format, as long as you give appropriate credit to the original author(s) and the source, provide a link to the Creative Commons license, and indicate if changes were made. The images or other third party material in this article are included in the article's Creative Commons license, unless indicated otherwise in a credit line to the material. If material is not included in the article's Creative Commons license and your intended use is not permitted by statutory regulation or exceeds the permitted use, you will need to obtain permission directly from the copyright holder. To view a copy of this license, visit http://creativecommons.org/ licenses/by/4.0/.

(C) The Author(s) 2021

${ }^{1}$ Chongqing Key Laboratory of Big Data for Bio Intelligence, Chongqing University of Posts and Telecommunications, Chongqing, China. ${ }^{2}$ European Molecular Biology Laboratory, European Bioinformatics Institute, Wellcome Genome Campus, Hinxton, UK. ${ }^{3}$ Algorithmic Bioinformatics, Freie Universität Berlin, Berlin, Germany. ${ }^{4}$ Visualization and Data analysis, Zuse Institute Berlin, Berlin, Germany. ${ }^{5}$ Moscow Institute of Physics and Technology, Dolgoprudny, Moscow Region, Russia. ${ }^{6}$ V.L. Talrose Institute for Energy Problems of Chemical Physics, N.N. Semenov Federal Research Center for Chemical Physics, Russian Academy of Sciences, Moscow, Russia. ${ }^{7}$ Bioinformatics Group Department of Computer Science, Albert-Ludwigs-University Freiburg, Freiburg, Germany. ${ }^{8}$ Institute for Surgical Pathology, Medical Center - University of Freiburg, Faculty of Medicine, University of Freiburg, Freiburg, Germany. ${ }^{9}$ Khoury College of Computer Sciences, Northeastern University, Boston, MA, USA. ${ }^{10}$ Department of Dermatology, Medical University of Vienna, Vienna, Austria. ${ }^{11}$ Department of Clinical Sciences, University of Bergen, Bergen, Norway. ${ }^{12}$ Department of Congenital Heart Disease and Pediatric Cardiology, Universitätsklinikum Schleswig-Holstein Kiel, Kiel, Germany. ${ }^{13}$ Novo Nordisk Foundation Center for Protein Research, University of Copenhagen, Copenhagen, Denmark. ${ }^{14}$ Ruhr University Bochum, Medical Faculty, Medizinisches Proteom-Center, 44801 Bochum, Germany. ${ }^{15}$ Ruhr University Bochum, Center for Protein Diagnostics (PRODI), Medical Proteome Analysis, 44801 Bochum, Germany. ${ }^{16}$ VIB - UGent Center for Medical Biotechnology, VIB, Ghent, Belgium. ${ }^{17}$ Department of Biomolecular Medicine, Faculty of Medicine and Health Sciences, Ghent University, Ghent, Belgium. ${ }^{18}$ Department of Biochemistry and Molecular Biology, University of Southern Denmark, Campusvej 55, 5230 Odense, Denmark. ${ }^{19}$ University of Pennsylvania, Department of Biology, Philadelphia, PA 19104, USA. ${ }^{20}$ Institute of Pharmacology and Structural Biology, University of Toulouse, CNRS, UPS, Toulouse, France. ${ }^{21}$ nference Labs, Bengaluru, KA 560017, India. ${ }^{22}$ Chair of Proteomics and Bioanalytics, Technical University of Munich, Munich, Germany. ${ }^{23}$ Department of Microchemistry, Proteomics and Lipidomics, Genentech, South San Francisco, CA, USA. ${ }^{24}$ Skaggs School of Pharmacy and Pharmaceutical Sciences, University of California San Diego, La Jolla, CA 92093, USA. ${ }^{25}$ Department of Computer Science, Applied Bioinformatics, University of Tübingen, Tübingen 72076, Germany. ${ }^{26}$ Institute for Biological and Medical Informatics, University of Tübingen, Tübingen 72076 , Germany. ${ }^{27}$ Institute for Translational Bioinformatics, University Hospital Tübingen, 72076 Tübingen, Germany. ${ }^{28}$ Center for Computational Mass Spectrometry, Department of Computer Science and Engineering, Skaggs School of Pharmacy and Pharmaceutical Sciences, University of California, San Diego, CA 92093-0404, USA. ${ }^{29}$ Institute for Systems Biology, 401 Terry Ave N, Seattle, WA 98109, USA. ${ }^{30}$ State Key Laboratory of Proteomics, Beijing Proteome Research Center, National Center for Protein Sciences (Beijing), Beijing Institute of Life Omics, Beijing 102206, China. 凶email: baimz@cqupt.edu.cn; timo.sachsenberg@uni-tuebingen.de; lev.levitsky@phystech.edu; yperez@ebi.ac.uk 